\title{
Design of Squirrel-Cage Self-Excited 3-phase Induction Generator
}

\author{
Bilal Abdullah Nasir
}

\begin{abstract}
Due to the wide utilization of a 3-phase, squirrel-cage, self-excitation generator in renewable energy and isolated areas application, the paper deals with detailed design procedure of this type of generator, in a sequence and systematic manner. The design steps are obtained and executed in a Matlab file of the computer program to suit the newly designed constructions and parameters of the generator. In the new construction, the generator can be operated in a stable and saturation region. Due to the similarity in the construction of induction motor and generator, the formulas used in the calculation of equivalent circuit components of an induction motor may be utilized to calculate the parameters of the induction generator. To obtain optimized induction generator parameters and construction dimensions an ant colony algorithm is used to optimize these construction dimensions and generator parameters. The main objective parameters used in this algorithm are the generator efficiency, the excitation-capacitance, winding temperature rise, and minimum generator size is for $5 \mathrm{HP}, 400 \mathrm{~V}, 50 \mathrm{~Hz}$, and star connection generator.
\end{abstract}

Keywords: Squirrel-Cage Induction Generator, Design Steps, Generator Parameters, Ant Colony Optimization.

\section{INTRODUCTION}

In recent years, the increasing demand for renewable energy has caused great interest in the development and utilization of wind and micro-hydro-electric power plants. Many papers showed the importance of wind and microhydro induction generators' performance analysis, modeling, protection, and control [1-5]. However, there are fewer publications that proposed the design and construction of these types of generators to develop an effective machine for electricity generation, especially in isolated areas and standalone generators [6-10]. Two types of secondary windings of the rotor in the induction generator are slip-ring wound rotor and squirrel-cage rotor. Squirrel-cage-type generators are used widely in isolated area applications. The major disadvantages of this type of generator are the low efficiency, high volume size, and low power factor. With a change in the design of the magnetic circuits of this type of generator, the performance can be improved.

Manuscript received on October 11, 2021.

Revised Manuscript received on October 16, 2021.

Manuscript published on October 30, 2021.

* Correspondence Author

Bilal A. Nasir*, Lecturer, Northern Technical University, Iraq.

(c) The Authors. Published by Blue Eyes Intelligence Engineering and Sciences Publication (BEIESP). This is an open access article under the CC BY-NC-ND license (http://creativecommons.org/licenses/by-nc-nd/4.0/)
These types of generators are used more than the other type of generators due to the ease of operation, construction, maintenance, and low noise. The design of the induction generator for desired and suitable performance must be not restricted by stator and rotor structure. The induction machine is operated as a magnetic circuit and it will be influenced by the magnetic saturation. However, the operation of the squirrel-cage self-excited induction generator becomes stable when the generator workes in the saturation region. Then, the best step to optimize the design of an induction generator is to design a new machine that can carry the saturated magnetizing current and handle the great terminal voltage. The similarity in the construction of induction motor and generator makes it possible to use the design steps of induction motor for the procedure of induction generator. The mathematical formulas and empirical equations used to calculate the equivalent circuit components of the induction motor can be used to calculate the components of the induction generator equivalent circuit. In this paper, a detailed design procedure of squirrelcage, self-excited induction generator is presented systematically with a design algorithm flow chart, by modifying the classical method of induction motor design. Recently, to solve the optimization requirements an ant colony algorithm has been suggested, which is derived from the foraging behavior of ants to optimize the designed parameters of induction machines [11-13]. In this paper, the ant colony optimization algorithm-based method is used for the optimal design of a squirrel-cage self-excited induction generator. In this method, optimal values of design parameters are determined to maximize the generator efficiency and to minimize the generator excitation capacitance, stator winding temperature, and generator volume.

\section{DESIGN STEPS}

The design procedure of the squirrel-cage, self-excited induction generator involves several steps. The sequence of the design steps can be summarized in a flowchart as shown in figure (1). These steps can be executed as follow:

1- The first step in the design of a squirrel-cage, self-excited induction generator is to define the fixed input specifications data of the generator. For example, the generator has $5 \mathrm{HP}$ output power, $400 \mathrm{~V}$ line voltage, $50 \mathrm{~Hz}$, 3-phase supply, and Y-connection type. These data can be states in table (1). 
Design of Squirrel-Cage Self-Excited 3-phase Induction Generator

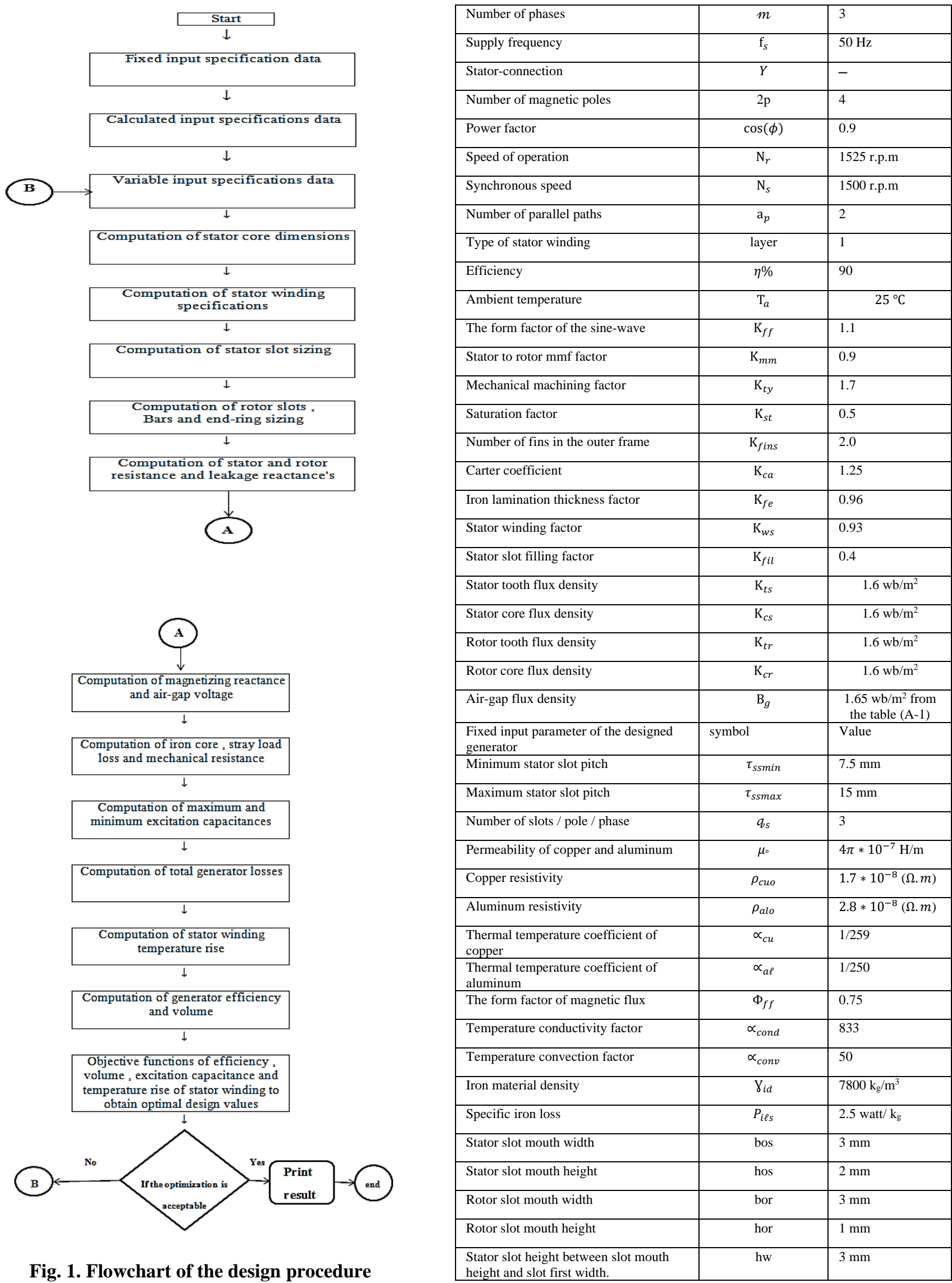

Table- 1: fixed input specifications

\begin{tabular}{|l|c|l|}
\hline $\begin{array}{c}\text { Input parameters of the designed } \\
\text { generator }\end{array}$ & Symbol & \multicolumn{1}{c|}{ Value } \\
\hline Output power & $\mathrm{P}_{\text {out }}$ & $5 \mathrm{HP}$ \\
\hline Phase voltage & $\mathrm{V}_{p h}$ & $230 \mathrm{~V}$ \\
\hline
\end{tabular}

Retrieval Number: 100.1/ijeat.A31981011121

DOI: 10.35940/ijeat.A3198.1011121

Journal Website: www.ijeat.org

Published By:

Blue Eyes Intelligence Engineering and Sciences Publication (BEIESP)

182 (C) Copyright: All rights reserved. calculation formula as shown in table (2).

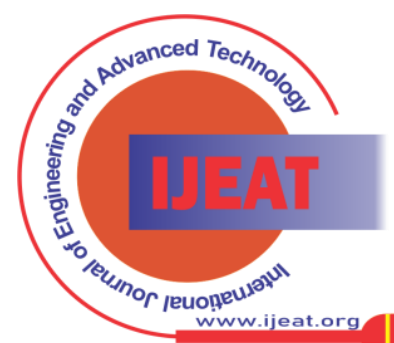


Table- 2: calculated input specifications data

\begin{tabular}{|l|c|lc|}
\hline $\begin{array}{l}\text { The calculated input } \\
\text { parameter }\end{array}$ & symbol & Calculation formula & \\
\hline Machine rated slip & $\mathrm{s}_{\mathrm{r}}$ & $\mathrm{s}_{\mathrm{r}}=\left(N_{s}-N_{r}\right) / N_{s}$ \\
\hline $\begin{array}{l}\text { Machine synchronous } \\
\text { speed }\end{array}$ & $n_{s}$ & $n_{s}=2 * f_{s} /(2 P) \quad(r . p . s)$ \\
\hline $\begin{array}{l}\text { Stator angular } \\
\text { frequency }\end{array}$ & $w_{s}$ & $w_{s}=2 * \pi * f_{s} \quad$ rad./sec. \\
\hline line-to-line voltage & $V_{\ell \ell}$ & $V_{\ell \ell}=\sqrt{3} * V_{p h} \quad$ (Volts) \\
\hline $\begin{array}{l}\text { Induced e.m.f } \\
\text { coefficient }\end{array}$ & $K_{e}$ & $K_{e}=0.98-0.005 * P$ \\
\hline
\end{tabular}

3- The variable input parameters as shown in table (3). These parameters can be changed in values by the computer program to obtain the optimal design.

Table- 3: variable input specifications data

\begin{tabular}{|c|c|c|}
\hline $\begin{array}{c}\text { Variable input } \\
\text { parameters }\end{array}$ & symbol & Value \\
\hline $\begin{array}{c}\text { Volume controlling } \\
\text { ratio }= \\
\frac{\text { stator stacklength }}{\text { pole pitch }}\end{array}$ & $\lambda_{v c}$ & 1.1 from the table (A-2) \\
\hline $\begin{array}{c}\text { Inner to outer stator } \\
\text { core diameter ratio }\end{array}$ & $K_{d s}$ & $0.62 \quad$ from the table (A-3) \\
\hline $\begin{array}{c}\text { Stator conductor } \\
\text { current density }\end{array}$ & $J_{c o}$ & $4 * 10^{6} \quad \mathrm{~A} / \mathrm{m}^{2}$ from the table (A-4) \\
\hline $\begin{array}{c}\text { Rotor bar current } \\
\text { density }\end{array}$ & $J_{b}$ & $5 * 10^{6} \quad \mathrm{~A} / \mathrm{m}^{2}$ \\
\hline $\begin{array}{c}\text { Rotor end-ring current } \\
\text { density }\end{array}$ & $J_{e r}$ & $25000 \quad(\mathrm{~A} / \mathrm{m})$ \\
\hline $\begin{array}{c}\text { Specific electrical } \\
\text { loading }\end{array}$ & $A_{c}$ & $500 \quad(\mathrm{~A}$. conductor $)$ \\
\hline $\begin{array}{c}\text { Specific stator slot } \\
\text { loading }\end{array}$ & $A_{s s}$ & \\
\hline
\end{tabular}

4- Calculation of stator core dimensions as shown in table (4). This table shows the related parameters to the stator core dimensions and their related calculated formulas.

Table- 4: stator core dimensions and their related formulas

\begin{tabular}{|c|c|c|}
\hline Parameter & symbol & Related formula \\
\hline $\begin{array}{l}\text { Apparent } \\
\text { generator power }\end{array}$ & $S_{g}$ & $S_{g}=P_{\text {out }} /(\eta * \cos \phi)$ \\
\hline $\begin{array}{l}\text { Generator } \\
\text { volume } \\
\text { utilization factor } \\
\text { or output } \\
\text { coefficient } \\
\end{array}$ & $C_{o}$ & $\begin{array}{l}C_{o}=K_{f f} * \phi_{f f} * K_{w s} * \pi * B_{g} * A_{c} \\
\ldots \ldots \text { Joule } / \mathrm{m}^{3}\end{array}$ \\
\hline $\begin{array}{l}\text { Stator bore } \\
\text { diameter }\end{array}$ & $D_{b s}$ & $\begin{array}{l}D_{b s}=\left[2 P * S_{g} /\left(C o * \pi * \lambda_{v c} * n_{s}\right)\right]^{\frac{1}{3}} \\
\ldots \ldots(\mathrm{mm})\end{array}$ \\
\hline $\begin{array}{l}\text { Stator outer } \\
\text { diameter }\end{array}$ & $D_{o s}$ & $D_{o s}=D_{b s} / K_{d s} \quad(\mathrm{~mm})$ \\
\hline $\begin{array}{l}\text { Stator stack } \\
\text { length }\end{array}$ & $\ell_{s s}$ & $\ell_{s s}=\left(\pi * \lambda_{v c} * D_{b s}\right) / 2 P$ \\
\hline $\begin{array}{l}\text { Stator pole- } \\
\text { pitch }\end{array}$ & $\tau_{p}$ & $\tau_{p}=\left(\pi * D_{b s}\right) / 2 P \quad(\mathrm{~mm})$ \\
\hline $\begin{array}{l}\text { Maximum stator } \\
\text { slot number }\end{array}$ & $Q_{\text {smax }}$ & $Q_{s \max }=\pi * D_{b s} / \tau_{s s \min } \quad(\mathrm{mm})$ \\
\hline $\begin{array}{l}\text { Minimum stator } \\
\text { slot number }\end{array}$ & $Q_{\text {smin }}$ & $Q_{\text {smin }}=\pi * D_{b s} / \tau_{\text {ssmax }} \quad(\mathrm{mm})$ \\
\hline $\begin{array}{l}\text { The suitable } \\
\text { number of stator } \\
\text { slots }\end{array}$ & $Q_{s}$ & From table (A-5) \\
\hline Stator slot pitch & $\tau_{S S}$ & $\tau_{s S}=\pi * D_{b s} / Q_{s} \quad(\mathrm{~mm})$ \\
\hline $\begin{array}{l}\text { Effective air- } \\
\text { gap length }\end{array}$ & $g_{e}$ & $\begin{array}{l}g_{e}=0.2+2 *\left[D_{b s} * \ell_{s s} * 10^{-6}\right]^{\frac{1}{2}} \\
(\mathrm{~mm})\end{array}$ \\
\hline
\end{tabular}

5- Stator winding calculation, as shown in table (5).
This table shows the parameters related to the stator winding and their calculated formulas.

Table- 5: stator winding calculations

\begin{tabular}{|l|c|l|}
\hline Parameters & Symbol & Related formula \\
\hline $\begin{array}{l}\text { Stator flux per } \\
\text { pole }\end{array}$ & $\Phi_{p}$ & $\Phi_{p}=\phi_{f f} * \tau_{p} * \ell_{s s} * B_{g}$ (weber) \\
\hline $\begin{array}{l}\text { Stator turns per } \\
\text { phase }\end{array}$ & $N_{p h}$ & $\begin{array}{l}N_{p h}= \\
V_{p h} /\left(4 * K_{e} * K_{f f} * F_{s} * \Phi_{p}\right)\end{array}$ \\
\hline $\begin{array}{l}\text { Stator conductors } \\
\text { per slot }\end{array}$ & $S_{s c}$ & $S_{s c}=2 * m * a_{p} * N_{p h} / Q_{s}$ \\
\hline $\begin{array}{l}\text { Stator load line } \\
\text { current }\end{array}$ & $I_{\ell \ell}$ & $\begin{array}{l}I_{\ell \ell}=P_{o u t} /\left(\eta * \sqrt{3} * V_{\ell \ell} * \cos \phi\right) \\
(\mathrm{A})\end{array}$ \\
\hline $\begin{array}{l}\text { Stator load phase } \\
\text { current }\end{array}$ & $I_{p h \ell}$ & $I_{p h \ell}=I_{\ell \ell}$ \\
\hline $\begin{array}{l}\text { Generator reactive } \\
\text { power }\end{array}$ & $Q_{g r}$ & $Q_{g r}=S_{g} * \sin (\phi) \quad$ (VAR) \\
\hline $\begin{array}{l}\text { Stator self- } \\
\text { excitation } \\
\text { capacitive } \\
\text { reactance }\end{array}$ & $X_{c p h}$ & $X_{c p h}=3 * V_{p h}^{2} / Q_{g r} \quad(\Omega)$ \\
\hline $\begin{array}{l}\text { Stator excitation } \\
\text { current }\end{array}$ & $I_{c p h}$ & $I_{c p h}=V_{p h} / X_{c p h}$ \\
\hline $\begin{array}{l}\text { Stator phase } \\
\text { current }\end{array}$ & $I_{s p h} \quad$ (A) \\
\hline $\begin{array}{l}\text { Stator specific slot } \\
\text { loading }\end{array}$ & $A_{s s}$ & $I_{s p h}=\left[I_{p h \ell}^{2}+I_{c p h}^{2}\right]^{\frac{1}{2}} \quad$ (A) \\
\hline $\begin{array}{l}\text { Stator winding } \\
\text { conductor cross- } \\
\text { sectional area }\end{array}$ & $A_{c o} * S_{s p h} \quad \ldots$. (A.cond.) \\
\hline $\begin{array}{l}\text { Stator winding } \\
\text { conductor } \\
\text { diameter }\end{array}$ & $A_{c o}=I_{s p h} / J_{c o} \quad$ (mm²) \\
\hline $\begin{array}{l}\text { Stator } \\
\text { Slot }\end{array}$ & $D_{c o}=4 * A_{c o} / \pi \quad$ (mm) \\
\hline
\end{tabular}

6- Stator slot sizing calculations as shown in table (6). This table shows the related parameters to the stator slots and their formulas of calculation. The stator slot structure is shown in figure (2).

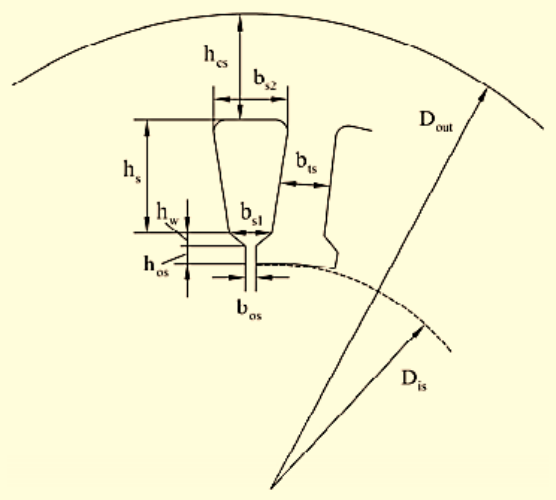

Fig. 2. Stator slot structure

Table- 6: Stator slot sizing calculations

\begin{tabular}{|c|c|c|}
\hline $\begin{array}{c}\text { Para } \\
\text { mete } \\
\mathrm{r}\end{array}$ & Symbol & Related calculation formula \\
\hline $\begin{array}{c}\text { Stato } \\
\mathrm{r} \\
\text { usefu } \\
\text { l slot } \\
\text { area }\end{array}$ & $A_{\text {use }}$ & $\begin{array}{c}A_{u s e}=A_{c o} * S_{s c} / K_{f i l} \\
K_{f i l} \text { can be obtained from the table (A-6) }\end{array}$ \\
\hline $\begin{array}{c}\text { Stato } \\
\mathrm{r} \\
\text { tooth } \\
\text { widt } \\
\mathrm{h}\end{array}$ & $b_{t s}$ & $b_{t s}=B_{g} * \tau_{s s} /\left(K_{t s} * K_{f e}\right)$ \\
\hline
\end{tabular}

Published By:

Blue Eyes Intelligence Engineering and Sciences Publication (BEIESP) 
Design of Squirrel-Cage Self-Excited 3-phase Induction Generator

\begin{tabular}{|c|c|c|}
\hline $\begin{array}{l}\text { Stato } \\
\text { r slot } \\
\text { widt } \\
\mathrm{h}(1)\end{array}$ & $b_{s 1}$ & $\begin{array}{c}b_{s 1}=\left[\pi *\left(D_{b s}+2 * h_{o s}+2 * h_{w}\right) / Q_{s}\right]-b_{t s} \\
\ldots \ldots \ldots\end{array}$ \\
\hline $\begin{array}{l}\text { Stato } \\
\text { r slot } \\
\text { widt } \\
\text { h (2) }\end{array}$ & $b_{s 2}$ & $b_{s 2}=\left[4 * A_{u s e} * \tan \left(\pi / Q_{s}+b_{s 1}^{2}\right)\right]^{\frac{1}{2}}(\mathrm{~mm})$ \\
\hline $\begin{array}{c}\text { Stato } \\
\text { r } \\
\text { usefu } \\
\text { l slot } \\
\text { heigh } \\
\mathrm{t} \\
\end{array}$ & $h_{s S}$ & $h_{s s}=2 * A_{u s e} / b_{s 1}+b_{s 2}$ \\
\hline $\begin{array}{c}\text { Stato } \\
\mathrm{r} \\
\text { back } \\
\text { iron } \\
\text { core } \\
\text { heigh } \\
\mathrm{t}\end{array}$ & $h_{c s}$ & $\begin{array}{c}h_{c s}=\left[D_{o c}-\left\{D_{b s}+2 *\left(h_{o s}+h_{w}+h_{s s}\right)\right\}\right] / 2 \\
(\mathrm{~mm})\end{array}$ \\
\hline
\end{tabular}

7- Calculation of rotor slots, bars, and end-ring sizing as shown in table (7).

This table shows the related formulas for the calculation of rotor slots, bars, and end-ring sizing. The rotor slot geometry is shown in figure (3).

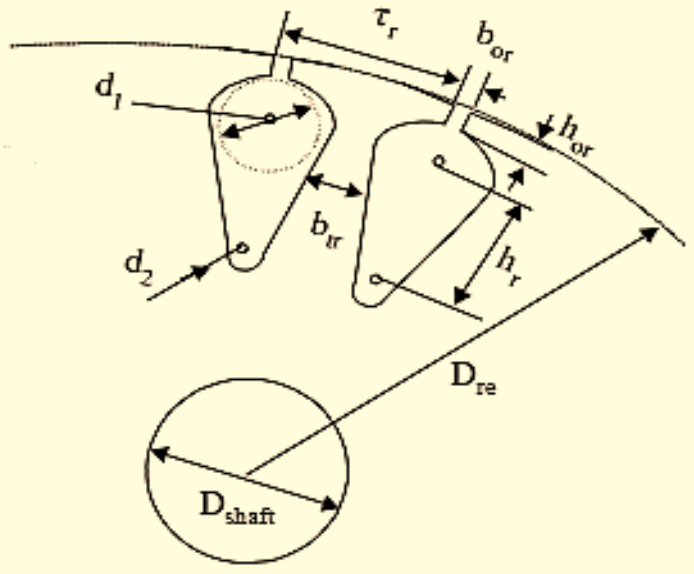

Fig. 3. Rotor slot structure

Table- 7: Rotor slots, bars, and end-ring sizing

\begin{tabular}{|c|c|c|}
\hline Parameter & $\begin{array}{c}\text { Symbo } \\
1\end{array}$ & Related calculation formula \\
\hline Number of rotor slots & $Q_{r}$ & From table (A-5) \\
\hline $\begin{array}{c}\text { Rotor current per } \\
\text { phase referred to } \\
\text { stator } \\
\end{array}$ & $\bar{I}_{r p h}$ & $\begin{array}{c}\dot{I}_{r p h}=I_{s p h} / K_{m m} \\
(\mathrm{~mm})\end{array}$ \\
\hline Rotor bar current & $I_{b}$ & $\begin{array}{c}I_{b}=2 * m * N_{p h} * K_{w s} * I_{r p h} / Q_{r} \\
(\mathrm{~A})\end{array}$ \\
\hline $\begin{array}{c}\text { Rotor slot area or } \\
\text { rotor bar area } \\
\end{array}$ & $A_{b}$ & $\begin{array}{c}A_{b}=I_{b} / J_{b} \\
\left(\mathrm{~mm}^{2}\right)\end{array}$ \\
\hline Rotor end-ring current & $I_{e r}$ & $I_{e r}=I_{b} /\left(2 * \sin \left(\pi * P / Q_{r}\right)\right)$ \\
\hline $\begin{array}{c}\text { Rotor end-ring cross- } \\
\text { sectional area } \\
\end{array}$ & $A_{\text {er }}$ & $\begin{array}{c}A_{e r}=I_{e r} / J_{e r} \\
\left(\mathrm{~mm}^{2}\right)\end{array}$ \\
\hline Rotor outer diameter & $D_{o r}$ & $\begin{array}{c}D_{o r}=\left(D_{b s}-2 * g_{e}\right) \\
(\mathrm{mm})\end{array}$ \\
\hline Rotor slot pitch & $\tau_{s r}$ & $\begin{array}{c}\tau_{s r}=\pi *=\left(\begin{array}{c}\left(D_{b s}-2 * g_{e}\right) / Q_{r} \\
(\mathrm{~mm})\end{array}\right. \\
\end{array}$ \\
\hline Rotor tooth width & $b_{t r}$ & $\begin{array}{c}b_{t r}=B_{g} * \tau_{s r} /\left(K_{f e} * K_{t r}\right) \\
(\mathrm{mm})\end{array}$ \\
\hline Rotor slot width & $b_{s r}$ & $\begin{aligned} b_{s r}= & \left(\tau_{s r}-b_{t r}\right) \\
& (\mathrm{mm})\end{aligned}$ \\
\hline $\begin{array}{l}\text { Rotor core height } \\
\text { (core back iron) }\end{array}$ & $h_{c r}$ & $\begin{array}{c}h_{c r}=\Phi_{p} /\left(2 * \ell_{s s} * K_{c r}\right) \\
(\mathrm{mm})\end{array}$ \\
\hline $\begin{array}{l}\text { Upper diameter of } \\
\text { rotor slot geometry }\end{array}$ & $d_{1}$ & $d_{1}=$ \\
\hline
\end{tabular}

\begin{tabular}{|c|c|c|}
\hline & & {$\left[\begin{array}{c}\pi *\left(D_{o r}-2 * h_{o r}\right)- \\
Q_{r} * b_{t r} \\
(\mathrm{~mm})\end{array}\right] /\left(\pi+Q_{r}\right)$} \\
\hline $\begin{array}{l}\text { Lower diameter of } \\
\text { rotor slot geometry }\left(d_{2}\right. \\
\left.\text { must be less than } d_{1}\right)\end{array}$ & $d_{2}$ & $\begin{array}{c}d_{2}= \\
\left\{\left(\begin{array}{c}32 * \\
A_{b} * \\
\tan \left(\pi / Q_{r}\right) \\
- \\
8+ \\
4 * \\
\pi * \\
\tan \left(\pi / Q_{r}\right) \\
* d_{1}^{2} \\
\ldots \ldots(\mathrm{mm})\end{array}\right) /\left(\begin{array}{c}4 * \\
\pi * \\
\tan \left(\pi / Q_{r}\right) \\
-8\end{array}\right.\right.\end{array}$ \\
\hline Rotor slot depth & $h_{s r}$ & $h_{s r}=\left(d_{1}-d_{2}\right) / 2 * \tan \left(\pi / Q_{r}\right)$ \\
\hline $\begin{array}{l}\text { Rotor end-ring height } \\
\text { (radial thickness) }\end{array}$ & $b$ & $\begin{array}{c}b=1.1 *\left(h_{s r}+h_{o r}+\left(d_{1}+\right.\right. \\
\left.\left.d_{2}\right) / 2\right)(\mathrm{mm})\end{array}$ \\
\hline $\begin{array}{l}\text { Rotor end-ring width } \\
\text { (axial thickness) }\end{array}$ & $a$ & $\begin{array}{c}a=A_{e r} / b \\
(\mathrm{~mm})\end{array}$ \\
\hline Rotor shaft diameter & $D_{s h}$ & $\begin{array}{c}D_{s h}=\left[D_{o r}-2 *\left(h_{o r}+\frac{\left(d_{1}+d_{2}\right)}{2}+\right.\right. \\
\left.\left.h_{s r}+h_{c r}\right)\right] \\
(\mathrm{mm})\end{array}$ \\
\hline
\end{tabular}

8- Calculation of stator resistance per phase as shown in table (8). This table shows all the parameters related to the calculation of stator resistance and their formulas.

Table- 8: calculation of stator resistance

\begin{tabular}{|l|c|l|}
\hline Parameter & Symbol & Related calculation formula \\
\hline $\begin{array}{l}\text { Number of stator slots } \\
\text { per pole }\end{array}$ & $P_{s}$ & $P_{s}=Q_{s} /(2 P)$ \\
\hline Chording factor & $K_{c h}$ & $K_{c h}=\cos \left(\pi /\left(2 * P_{s}\right)\right)$ \\
\hline Coil span & $y_{s c}$ & $\begin{array}{l}y_{s c}=\tau_{p} * K_{c h} \\
(\mathrm{~mm})\end{array}$ \\
\hline $\begin{array}{l}\text { Length of end-turn from } \\
\text { the table (A-7) }\end{array}$ & $\ell_{e t}$ & $\begin{array}{l}\ell_{e t}=\left(y_{s c}+20 * 10^{-3}\right) \\
(\mathrm{mm})\end{array}$ \\
\hline Length of one turn & $\ell_{t}$ & $\begin{array}{l}\ell_{t}=2 *\left(\ell_{s s}+\ell_{e t}\right) \\
(\mathrm{mm})\end{array}$ \\
\hline $\begin{array}{l}\text { Stator resistance per } \\
\text { phase }\end{array}$ & $R_{s}$ & $\begin{array}{l}R_{s}=\rho_{c u o} * \ell_{t} * N_{p h} /\left(A_{c o} *\right. \\
\left.a_{p}\right)(\Omega)\end{array}$ \\
\hline
\end{tabular}

9- Calculation of rotor resistance per phase referred to stator side as shown in table (9). This table shows all the parameters related to the calculation of rotor phase resistance and their formulas.

Table- 9: calculation of rotor phase resistance

\begin{tabular}{|l|c|l|}
\hline Parameter & Symbol & Related calculation formula \\
\hline $\begin{array}{l}\text { Length of } \\
\text { end-ring per } \\
\text { rotor slot }\end{array}$ & $\ell_{\text {ers }}$ & $\begin{array}{l}\ell_{\text {ers }}=\pi *\left(D_{\text {or }}-2 * h_{s r}-b\right) / Q_{r} \\
(\mathrm{~mm})\end{array}$ \\
\hline $\begin{array}{l}\text { Skin effect } \\
\text { factor }\end{array}$ & $K_{r}$ & $\begin{array}{l}K_{r}=h_{s r} *\left[2 * \pi * f_{r} * \mu_{o} /\left(2 * \rho_{\text {alo }}\right)\right]^{\frac{1}{2}} \\
\text { Where } f_{r}=S \cdot f_{s} \text { rotor voltage frequency }\end{array}$ \\
\hline $\begin{array}{l}\text { Skew effect } \\
\text { factor }\end{array}$ & $K_{s q}$ & $K_{s q}=\sin \left(\pi * P / Q_{r}\right) /\left(\pi * P / Q_{r}\right)$ \\
\hline $\begin{array}{l}\text { Rotor } \\
\text { resistance } \\
\text { per phase }\end{array}$ & $R_{r}$ & $\begin{array}{l}R_{r}=\left[\left\{\rho_{c u o} * \ell_{s s} * K_{r} / A_{b}\right\}+\right. \\
\left.\left\{\rho_{\text {alo }} * \ell_{\text {ers }} /\left(2 * A_{\text {er }} * \sin ^{2}\left(\pi P / Q_{r}\right)\right)\right\}\right]\end{array}$ \\
\hline $\begin{array}{l}\text { Rotor } \\
\text { resistance } \\
\text { per phase } \\
\text { referred to } \\
\text { the stator } \\
\text { side }\end{array}$ & $R_{r}$ & $\dot{R}_{r}=\left[4 * m *\left(N_{p h} * K_{w s}\right)^{2} /\left(Q_{r} * K_{s q}^{2}\right)\right]$ \\
\hline
\end{tabular}

10- Calculation of stator leakage reactance per phase as shown in table (10).

Published By:

Blue Eyes Intelligence Engineering and Sciences Publication (BEIESP)

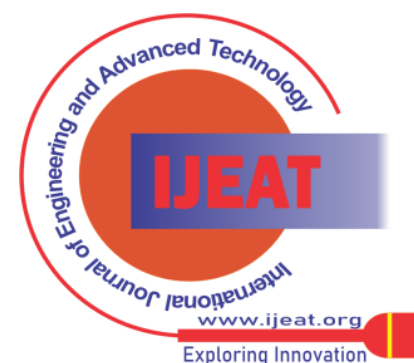


This table shows the parameters calculation steps with their formulas, which are used to calculate the stator leakage reactance.

Table- 10: calculation of stator leakage reactance

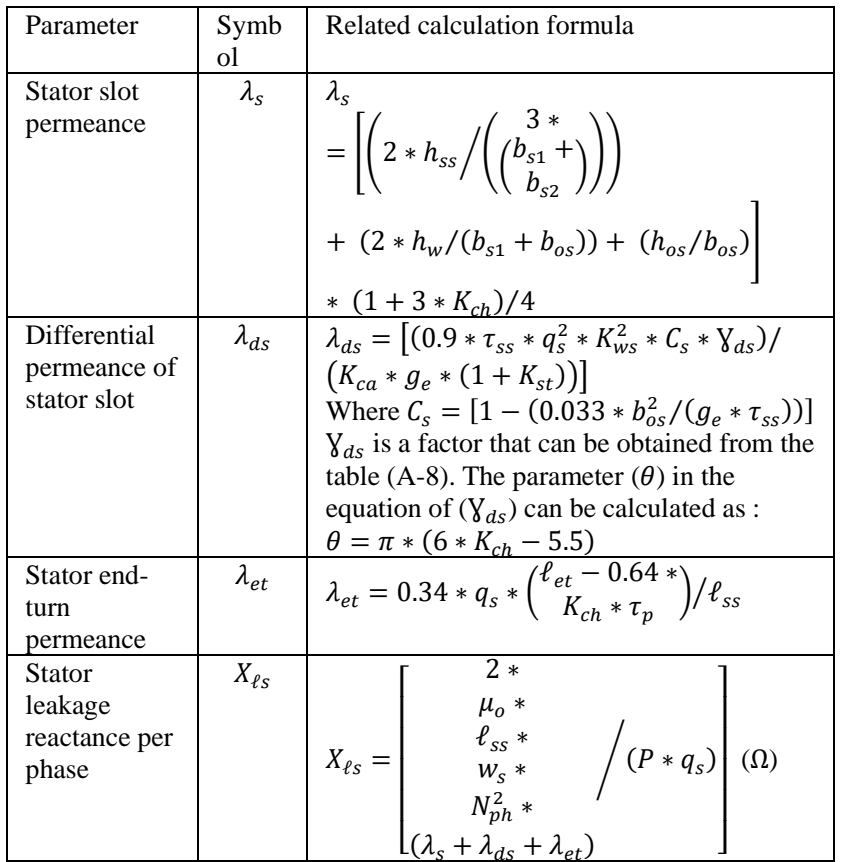

11- Calculation of rotor leakage reactance per phase as shown in table (11). The table involves all the parameters related to the rotor leakage reactance calculation with their formulas.

Table- 11: calculation of rotor leakage reactance

\begin{tabular}{|c|c|c|}
\hline Parameters & Symbol & Relate formula of calculation \\
\hline $\begin{array}{l}\text { Rotor slot } \\
\text { permeance }\end{array}$ & $\lambda_{r}$ & $\begin{aligned} \lambda_{r}=[0.66+(2 * h s r & /(3 \\
& \left.\left.*\left(d_{1}+d_{2}\right)\right)\right) \\
& \left.+h_{o r} / b_{o r}\right]\end{aligned}$ \\
\hline $\begin{array}{l}\text { Rotor slot } \\
\text { differential } \\
\text { permeance }\end{array}$ & $\lambda_{d r}$ & $\begin{array}{l}\lambda_{d r}=0.9 * \tau_{s r} * \gamma_{d r} * Q_{r}^{2} /\left(K_{c a} *\right. \\
\left.g_{e}(6 * P)^{2}\right) \\
\text { Where } \gamma_{d r}=9 *\left(6 * P / Q_{r}\right)^{2} * 10^{-2} \\
K_{c a}=\text { can be obtained from the table } \\
(\mathrm{A}-9) .\end{array}$ \\
\hline $\begin{array}{l}\text { Rotor end-ring } \\
\text { permeance }\end{array}$ & $\lambda_{e r}$ & $\begin{array}{l}\lambda_{e r}=\left[2.3 *\left(D_{o r}-b\right)\left(Q_{r} * \ell_{s s} * 4 *\right.\right. \\
\left.\left.\sin ^{2}\left(\pi * P / Q_{r}\right)\right)\right] * \log [4.7 * \\
\left.\left(D_{o r}-b\right) /(b+2 . a)\right]\end{array}$ \\
\hline $\begin{array}{l}\text { Skin-effect } \\
\text { factor }\end{array}$ & $K_{x}$ & $\begin{array}{l}K_{x}= \\
3 /\left[\begin{array}{c}2 * h_{s r} * \\
\left\{w_{s} * \mu_{o} /\left(2 * \rho_{a \ell o}\right)\right\}^{\frac{1}{2}}\end{array}\right] * 10^{-3}\end{array}$ \\
\hline $\begin{array}{l}\text { Rotor leakage } \\
\text { reactance per } \\
\text { phase }\end{array}$ & $X_{\ell r}$ & $\begin{array}{l}X_{\ell r}=w_{s} * \mu_{o} * \ell_{s s} *\left(\lambda_{r} * K_{x}+\right. \\
\left.\lambda_{d r}+\lambda_{e r}\right)\end{array}$ \\
\hline $\begin{array}{l}\text { Rotor leakage } \\
\text { reactance per } \\
\text { phase referred to } \\
\text { the stator side }\end{array}$ & $\dot{X}_{\ell r}$ & $\begin{array}{l}\dot{X}_{\ell r}=\left[4 * m *\left(N_{p h} * K_{w s}\right)^{2} /\left(Q_{r} *\right.\right. \\
\left.\left.K_{s q}^{2}\right)\right] * X_{\ell r}\end{array}$ \\
\hline
\end{tabular}

12- Calculation of magnetizing reactance per phase and the corrected rotor leakage reactance due to the skew effect as shown in table (12).

Table- 12: calculation of magnetizing reactance and the corrected rotor leakage reactance

\begin{tabular}{|c|c|c|}
\hline Parameters & Symbol & Related calculation formula \\
\hline $\begin{array}{l}\text { Magnetizing reactance } \\
\text { per phase }\end{array}$ & $X_{m}$ & $\begin{array}{l}X_{m}=w_{s} * K_{s t} * \mu_{o} * m * \tau_{p} * \\
\ell_{s s} *\left(N_{p h} * K_{w s}\right)^{2} /(\pi * P * \\
\left.g_{e}\right) \quad(\Omega)\end{array}$ \\
\hline $\begin{array}{l}\text { Rotor leakage reactance } \\
\text { due to skew-effect }\end{array}$ & $\dot{X}_{\text {lrsq }}$ & $\dot{X}_{\ell r s q}=X_{m} *\left(1-K_{s q}^{2}\right)$ \\
\hline $\begin{array}{l}\text { Total rotor leakage } \\
\text { reactance per phase }\end{array}$ & $\dot{X}_{\ell r t}$ & $\begin{array}{l}\dot{X}_{\ell r t}=\left(\dot{X}_{\ell r}+\dot{X}_{\text {lrsq }}\right) \\
(\Omega)\end{array}$ \\
\hline
\end{tabular}

Retrieval Number: 100.1/ijeat.A31981011121

DOI: 10.35940/ijeat.A3198.1011121

Journal Website: www.ijeat.org
13- Calculation of magnetizing voltage and no-load current per phase as shown in table (13).

Table- 13: calculation of magnetizing voltage and noload current

\begin{tabular}{|l|c|l|}
\hline Parameters & Symbol & Related calculation formula \\
\hline $\begin{array}{l}\text { Magnetizing or air-gap } \\
\text { phase voltage }\end{array}$ & $V_{g}$ & $\begin{array}{l}V_{g}=V_{p h}+I_{s p h} *\left[R_{s}^{2}+X_{\ell s}^{2}\right]^{\frac{1}{2}} \\
(\mathrm{~V})\end{array}$ \\
\hline No-load current / phase & $I_{o}$ & $\begin{array}{l}I_{o}=\left[\hat{I}_{r}^{2}-I_{s p h}^{2}\right]^{\frac{1}{2}} \\
\text { (A) }\end{array}$ \\
\hline Rotor phase current & $I_{r p h}$ & $\begin{array}{l}\hat{I}_{r p h}=V_{g} / \dot{Z}_{r} \\
\text { (A) }\end{array}$ \\
\hline $\begin{array}{l}\text { Magnetizing current per } \\
\text { phase }\end{array}$ & $I_{m}$ & $\begin{array}{l}I_{m}=V_{g} / X_{m} \\
\text { (A) }\end{array}$ \\
\hline
\end{tabular}

14- Calculation of maximum and minimum excitation capacitance of the generator as shown in table (14). The maximum and minimum capacitance per phase can be calculated from the equivalent circuit of the generator.

Table- 14: maximum and minimum excitation capacitance per phase

\begin{tabular}{|c|c|c|}
\hline Parameters & Symbol & Related calculation formula \\
\hline $\begin{array}{l}\text { Stator load loss } \\
\text { resistance [5] }\end{array}$ & $R_{\text {sle }}$ & $R_{s \ell \ell}=R_{S} * X_{\ell s}^{2} /\left[R_{S}^{2}+X_{\ell S}^{2}\right] \quad(\Omega)$ \\
\hline $\begin{array}{l}\text { Slip at } \\
\text { maximum torque }\end{array}$ & $S_{\max }$ & $\begin{array}{l}S_{\max }= \\
\dot{R}_{r} /\left[\left(R_{s}+R_{s \ell \ell}\right)^{2}+\left(X_{\ell s}+\dot{X}_{\ell r}\right)^{2}\right]^{\frac{1}{2}}\end{array}$ \\
\hline $\begin{array}{l}\text { Minimum rotor } \\
\text { speed }\end{array}$ & $W_{\text {rmin }}$ & $W_{\text {rmin }}=w_{s} *\left(1-S_{\max }\right) \quad \frac{\text { ele.rad }}{\text { sec. }}$ \\
\hline $\begin{array}{l}\text { Maximum rotor } \\
\text { speed }\end{array}$ & $W_{\operatorname{rmax}}$ & $W_{\text {rmax }}=w_{s} *\left(1+S_{\max }\right) \quad \frac{\text { ele.rad }}{\text { sec. }}$ \\
\hline $\begin{array}{l}\text { Maximum } \\
\text { excitation } \\
\text { capacitance per } \\
\text { phase }\end{array}$ & $C_{\text {emax }}$ & $\begin{array}{l}C_{\text {emax }}=1 /\left(W_{r \min }^{2} *\left(X_{m}+X_{\ell s}\right) / w_{s}\right) \\
(\mu F)\end{array}$ \\
\hline $\begin{array}{l}\text { Minimum } \\
\text { excitation } \\
\text { capacitance per } \\
\text { phase }\end{array}$ & $C_{e m i n}$ & $\begin{array}{l}C_{e \min }=1 /\left(W_{r \max }^{2} *\left(X_{m}+X_{\ell s}\right) / w_{s}\right) \\
(\mu F)\end{array}$ \\
\hline
\end{tabular}

15- Calculation of generator losses as shown in table (15).In this table, the details of losses calculation with their formulas are presented and then added to determine the total machine losses.

Table- 15: Generator losses calculation

\begin{tabular}{|c|c|c|}
\hline Parameters & Symbol & Related calculation formula \\
\hline $\begin{array}{l}\text { Stator copper } \\
\text { loss }\end{array}$ & $P_{\text {cus }}$ & $\begin{array}{l}P_{c u s}=3 * I_{s p h}^{2} * R_{s} \\
\text { (watt) }\end{array}$ \\
\hline $\begin{array}{l}\text { Rotor copper } \\
\text { loss }\end{array}$ & $P_{\text {cur }}$ & $\begin{array}{l}P_{\text {cur }}=3 * \hat{I}_{r}^{2} * \hat{R}_{r} \\
\text { (watt) }\end{array}$ \\
\hline Mechanical loss & $P_{\text {mec }}$ & $\begin{array}{l}P_{m e c}=0.012 * P_{\text {out }} \text { from the table } \\
(\mathrm{A}-10) \text { (watt) }\end{array}$ \\
\hline Iron core losses & $P_{i c}$ & $\begin{array}{l}P_{i c}=3 * V_{g} * I_{i c} \\
\text { (watt) } \\
\text { Where } I_{i c}=I_{o} * \cos \phi_{o}\end{array}$ \\
\hline Stray load losses & $P_{\text {sle }}$ & $P_{s \ell \ell}=3 * I_{s p h}^{2} * R_{s \ell \ell} \quad$ (watt) \\
\hline $\begin{array}{l}\text { Total machine } \\
\text { losses }\end{array}$ & $P_{t \ell}$ & $P_{t \ell}=P_{c u s}+P_{c u r}+P_{m e c}+P_{i c}+P_{s \ell \ell}$ \\
\hline
\end{tabular}

16- Calculation of generator temperature rise as shown in table (16).The table shows the stator and rotor winding resistivity calculation in terms of winding temperature rise.

Published By:

Blue Eyes Intelligence Engineering and Sciences Publication (BEIESP)

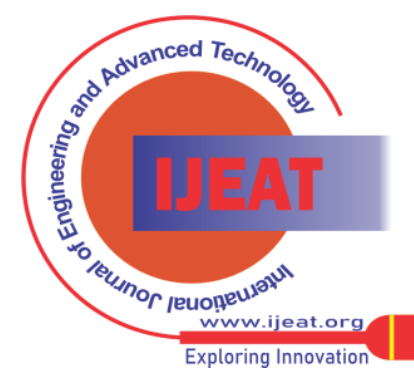


Table- 16: temperature rise calculation of the stator winding

\begin{tabular}{|c|c|c|}
\hline Parameters & Symbol & Related calculation formula \\
\hline $\begin{array}{l}\text { Machine frame } \\
\text { area }\end{array}$ & $A_{\text {frame }}$ & $\begin{array}{l}A_{\text {frame }}=\pi * D_{o s} *\left(\ell_{s s}+\tau_{p}\right) * \\
K_{\text {fins }} * 10^{-6} \\
\left(\mathrm{~m}^{2}\right)\end{array}$ \\
\hline Frame temperature & $T_{\text {frame }}$ & $\begin{array}{l}T_{\text {frame }}=P_{\text {t }} /\left(\propto_{\text {conv }} * A_{\text {frame }}\right)\left({ }^{\circ} \mathrm{C}\right) \\
\text { where } \propto_{\text {conv }}=50 \text { from table }(\mathrm{A}-11)\end{array}$ \\
\hline $\begin{array}{l}\text { Stator winding } \\
\text { temperature }\end{array}$ & $T_{\text {winding }}$ & $\begin{array}{c}T_{\text {winding }}=T_{\text {ambient }}+T_{\text {frame }} \quad\left({ }^{\circ} \mathrm{C}\right) \\
T_{\text {ambient }}=25^{\circ} \mathrm{C}\end{array}$ \\
\hline $\begin{array}{l}\text { The resistivity of } \\
\text { the stator winding }\end{array}$ & $\rho_{c u}$ & $\begin{array}{l}\rho_{c u}=\left(1+\propto_{c u} * T_{\text {winding }}\right) * \rho_{\text {cuo }} \\
(\Omega . m)\end{array}$ \\
\hline $\begin{array}{l}\text { The resistivity of } \\
\text { rotor bars }\end{array}$ & $\rho_{a \ell}$ & $\begin{array}{c}\rho_{a \ell}=\left(1+\propto_{a \ell} *\left(T_{\text {winding }}+10\right)\right) * \\
\rho_{a \ell o}\end{array}$ \\
\hline
\end{tabular}

17- Calculation of optimized parameters of the generator as shown in table (17).The main parameters that are optimized in this design of the induction generator are efficiency, excitation-capacitance, stator winding temperature, and the generator volume.

Table- 17: optimized parameters calculation

\begin{tabular}{|c|c|c|}
\hline parameters & Symbol & Related calculation formula \\
\hline $\begin{array}{l}\text { Generator } \\
\text { efficiency }\end{array}$ & $\eta \%$ & $\begin{array}{l}\eta \%=\left[P_{\text {out }} /\left(P_{\text {out }}+P_{\text {te }}\right)\right] * \\
100\end{array}$ \\
\hline $\begin{array}{l}\text { Excitation } \\
\text { capacitance }\end{array}$ & $C_{\text {emin }}$ & $\begin{array}{l}C_{e \min }= \\
1 /\left[w_{r \max }^{2} *\left(X_{m}+X_{\ell s}\right) / W_{s}\right]\end{array}$ \\
\hline $\begin{array}{l}\text { Stator winding } \\
\text { temperature }\end{array}$ & $T_{\text {winding }}$ & $T_{\text {winding }}=T_{\text {ambient }}+T_{\text {frame }}$ \\
\hline $\begin{array}{l}\text { Generator } \\
\text { volume }\end{array}$ & Volume & $\begin{array}{l}\text { Volume }=\left(\pi * D_{o s}^{2} * \ell_{s s} / 4\right) * \\
10^{-3}\left(C_{m}^{3}\right)\end{array}$ \\
\hline
\end{tabular}

\section{ANT COLONY OPTIMIZATION}

In this paper, for example, a 5-HP squirrel-cage, self-excited induction generator, $50 \mathrm{~Hz}$ and $400 \mathrm{~V}$ line-to-line is designed and optimized. The objective function is used for maximum efficiency, minimum excitation capacitance, minimum generator volume, and minimum temperature rise. The ant colony algorithm parameters are given as [12] :

$F_{(x, w)}=W_{1} * \frac{1}{[1+\eta]}+W_{2} * C_{\text {emin }}+W_{3} *$ volume $+W_{4} *$

$T_{\text {winding }}$

Where $W_{1}, W_{2}, W_{3}$ and $W_{4}$ are the weights that indicate the relative significance among the chosen objectives. These weight parameters can be set to one for activating the objective function. The multi-objective optimization problem can be suited by combining many objectives through weight parameters into a single objective to optimize the chosen objectives ( involves both minimizations and maximization ) simultaneously.

\section{RESULTS AND DISCUSSION}

The ant colony optimization algorithm is used to obtain the optimal design parameters of the squirrel-cage, self-excited induction generator.The design equations in tables (2-17) are used to feed the computer program in a sequence and systematic arrangement of the design procedure. The software package is developed and executed in a Matlab file. The final machine design results are obtained by optimizing the objectives of efficiency, excitation capacitance, machine volume, and stator winding temperature rise. It is clear from tables (18-19), which are the results of the design, those better design parameters are obtained when the weights $W_{1}=W_{2}=W_{3}=W_{4}=1$ in the ant colony algorithm of optimization. Table (18) shows the results of all machine construction parameters, while table (19) shows the optimal results of objective parameters. The algorithm offers maximum machine efficiency and minimum excitation capacitance, minimum size, and minimum stator winding temperature rise. In this case study, the machine efficiency is $88 \%$, the minimum excitation capacitance per phase is $25 \mu \mathrm{F}$, the minimum volume of the machine is $2260 C_{m}^{3}$ and the minimum stator winding temperature rise is $50^{\circ} \mathrm{C}$.

\section{Table- 18: Design specifications of the generator construction parameters}

\begin{tabular}{|c|c|c|}
\hline Generator construction parameters & Symbol & Dimension \\
\hline Stator stack length/pole pitch & $\lambda_{v c}$ & 1.15 \\
\hline Inner to outer stator core diameter & $K_{d s}$ & 0.625 \\
\hline Stator conductor current density & $J_{c o}$ & $4.5 * 10^{6}$ \\
\hline Rotor bar current density & $J_{b}$ & $4.5 * 10^{6}$ \\
\hline Rotor end-ring current density & $J_{e r}$ & $5.5 * 10^{6} \quad \mathrm{~A} / \mathrm{m}^{2}$ \\
\hline Specific electrical loading & $A_{c}$ & $23500 \mathrm{~A} / \mathrm{m}$ \\
\hline Specific stator slot loading & $A_{S S}$ & 450 A. conductor \\
\hline Apparent generator power & $S_{g}$ & $4725 \quad$ VA \\
\hline Generator volume utilization factor & $C_{o}$ & 35900 Joule $/ \mathrm{m}^{3}$ \\
\hline Stator outer diameter & $D_{o S}$ & $57 \mathrm{~mm}$ \\
\hline Stator stack length & $\ell_{S S}$ & $195 \mathrm{~mm}$ \\
\hline Stator pole-pitch & $\tau_{p}$ & $44.5 \mathrm{~mm}$ \\
\hline Stator slot number & $Q_{S}$ & 24 \\
\hline Effective air-gap length & $g_{e}$ & $1.12 \mathrm{~mm}$ \\
\hline Stator flux per pole & $\phi_{p}$ & 0.013 weber \\
\hline Stator turns per phase & $N_{p h}$ & 165 \\
\hline Stator phase current & $I_{\text {sph }}$ & 7.0 \\
\hline Stator winding conductor area & $A_{c o}$ & $\mathrm{~mm}^{2}$ \\
\hline Stator winding conductor diameter & $D_{c o}$ & $1.4 \mathrm{~mm}$ \\
\hline Stator useful slot area & $A_{\text {use }}$ & $21.0 \mathrm{~mm}^{2}$ \\
\hline Stator tooth width & $b_{t s}$ & $3.0 \quad \mathrm{~mm}$ \\
\hline Stator slot width (1) & $b_{s 1}$ & $\mathrm{~mm}$ \\
\hline Stator slot width (2) & $b_{2}$ & $2.5 \mathrm{~mm}$ \\
\hline Stator useful slot height & $h_{s S}$ & $15.0 \mathrm{~mm}$ \\
\hline Stator back-iron core height & $h_{c s}$ & $16.0 \mathrm{~mm}$ \\
\hline Rotor slot number & $Q_{r}$ & 22 \\
\hline Rotor phase current & $I_{r p h}$ & 7.3 \\
\hline Rotor bar current & $I_{b}$ & $164 \mathrm{~A}$ \\
\hline Rotor slot area & $A_{b}$ & $36.5 \mathrm{~mm}^{2}$ \\
\hline Rotor end-ring current & $I_{e r}$ & $264 \quad \mathrm{~A}$ \\
\hline Rotor end-ring cross-sectional area & $A_{e r}$ & $48 \quad \mathrm{~mm}^{2}$ \\
\hline Rotor outer diameter & $D_{o r}$ & $54.5 \mathrm{~mm}$ \\
\hline Rotor slot pitch & $\tau_{s r}$ & $\mathrm{~mm}$ \\
\hline Rotor tooth width & $b_{t r}$ & $\mathrm{~mm}$ \\
\hline Rotor slot width & $b_{s r}$ & $\mathrm{~mm}$ \\
\hline Rotor core height & $h_{c r}$ & $11.0 \mathrm{~mm}$ \\
\hline Rotor upper slot diameter & $d_{1}$ & $\mathrm{~mm}$ \\
\hline Rotor lower slot diameter & $d_{2}$ & 1.0 \\
\hline Rotor slot depth & $h_{s r}$ & $\mathrm{~mm}$ \\
\hline Rotor end-ring height & $b$ & $\mathrm{~mm}$ \\
\hline Rotor end-ring width & $a$ & $\mathrm{~mm}$ \\
\hline Rotor shaft diameter & $D_{s h}$ & $33.1 \mathrm{~mm}$ \\
\hline Stator resistance per phase & $R_{S}$ & $3.1 \Omega$ \\
\hline Rotor resistance per phase & $\dot{R}_{r}$ & $2.5 \Omega$ \\
\hline Stator leakage reactance per phase & $X_{\ell s}$ & $1.8 \Omega$ \\
\hline Rotor leakage reactance per phase & $\dot{X}_{\ell r}$ & $0.85 \Omega$ \\
\hline Magnetizing reactance per phase & $X_{m}$ & $140 \Omega$ \\
\hline $\begin{array}{l}\text { Magnetizing or air-gap voltage / } \\
\text { phase }\end{array}$ & $V_{g}$ & $250 \Omega$ \\
\hline No-load current per phase & $I_{O}$ & 2.0 \\
\hline Magnetizing current per phase & $I_{m}$ & 1.8 \\
\hline Iron core current per phase & $I_{\text {ic }}$ & 0.9 \\
\hline Iron core resistance per phase & $R_{i c}$ & 280 \\
\hline
\end{tabular}


Table- 19: objective design parameters of the generator

\begin{tabular}{|l|c|l|}
\hline Parameters & Symbol & Dimension \\
\hline $\begin{array}{l}\text { Minimum excitation } \\
\text { capacitance per phase }\end{array}$ & $C_{\text {emin }}$ & $25 \mu \mathrm{F}$ \\
\hline $\begin{array}{l}\text { Stator winding } \\
\text { temperature }\end{array}$ & $T_{\text {winding }}$ & $52{ }^{\circ} \mathrm{C}$ \\
\hline Generator volume & Volume & $2270 C_{m}^{3}$ \\
\hline Generator efficiency & Efficiency & $88 \%$ \\
\hline
\end{tabular}

\section{CONCLUSION}

Design steps with their suitable theoretical and empirical formulas are executed in the computer program and Matlab file to obtain the structure dimensions and parameters of the 3 -phase squirrel-cage self-excited induction generator. The design steps are summarized in sequence tables and systematic manner. The new design dimensions and parameters of the generator suit the generator operation in the stable and saturation region. The design results are optimized by an ant colony optimization algorithm. The objective parameters are used in this algorithm to obtain maximum generator efficiency and minimum excitation capacitance, minimum winding temperature rise, and minimum generator size. A generator of $5 \mathrm{HP}, 400 \mathrm{~V}, 50$ $\mathrm{Hz}$, and $\mathrm{Y}$-connection is used as an example of the design procedure.

\section{APPENDIX [14]}

Table- A-1: Air-gap flux density as a function of number of magnetic poles

\begin{tabular}{|l|l|l|l|l|}
\hline Poles Number (2P) & 2 & 4 & 6 & 8 \\
\hline Air-gap density & $0.5-$ & $0.65-0.78$ & $0.7-$ & $0.75-$ \\
$\left(\mathrm{wb} / \mathrm{m}^{2}\right)$ & 0.75 & & 0.82 & 0.85 \\
\hline
\end{tabular}

Table- A-2: Volume control parameter $\left(\lambda_{v c}\right)$ as a function of the number of poles

\begin{tabular}{|l|l|l|l|l|}
\hline Poles Number (2P) & 2 & 4 & 6 & 8 \\
\hline Volume controlling ratio & $0.6-$ & $1.2-1.8$ & $1.6-$ & $2-8$ \\
$\left(\lambda_{v c}\right)$ & 1.0 & & 2.2 & \\
\hline
\end{tabular}

Table- A-3: Stator inner to outer diameter ratio $\left(K_{d s}\right)$ as a function of poles number

\begin{tabular}{|l|l|l|l|l|}
\hline $\begin{array}{l}\text { Poles Number } \\
(2 \mathrm{P})\end{array}$ & 2 & 4 & 6 & 8 \\
\hline Ratio $\left(K_{d s}\right)$ & $0.54-$ & $0.61-$ & $0.68-$ & $0.72-0.74$ \\
& 0.58 & 0.68 & 0.71 & \\
\hline
\end{tabular}

Table- A-4: current density of stator winding as a function of poles number

\begin{tabular}{|l|l|l|}
\hline Poles Number (2P) & 2 or 4 & 6 or 8 \\
\hline $\begin{array}{l}\text { A current density of stator } \\
\text { winding }\left(U_{c o}\right)\end{array}$ & $4-7 \mathrm{~A} / \mathrm{mm}^{2}$ & $5-8 \mathrm{~A} / \mathrm{mm}^{2}$ \\
\hline
\end{tabular}

Table- A-5: stator and rotor slot number as a function of poles number

\begin{tabular}{|c|c|c|c|c|c|}
\hline$(2 \mathrm{P})$ & $Q_{S}$ & 24 & 36 & 48 & - \\
\hline 2 & $Q_{r}$ & $\begin{array}{l}18 \\
20\end{array}$ & $\begin{array}{l}32 \\
30 \\
28\end{array}$ & $\begin{array}{l}44 \\
40 \\
30\end{array}$ & \\
\hline & $Q_{S}$ & 24 & 36 & 48 & 72 \\
\hline 4 & $Q_{r}$ & $\begin{array}{l}22 \\
18\end{array}$ & $\begin{array}{l}34 \\
30 \\
28\end{array}$ & $\begin{array}{l}46 \\
42 \\
36 \\
\end{array}$ & $\begin{array}{l}70 \\
66 \\
62 \\
\end{array}$ \\
\hline & $Q_{S}$ & - & 36 & 54 & 72 \\
\hline 6 & $Q_{r}$ & & $\begin{array}{l}32 \\
28 \\
22\end{array}$ & $\begin{array}{l}46 \\
44 \\
40\end{array}$ & $\begin{array}{l}68 \\
64 \\
62\end{array}$ \\
\hline
\end{tabular}

Retrieval Number: 100.1/ijeat.A31981011121

DOI: 10.35940/ijeat.A3198.1011121

Journal Website: www.ijeat.org

\begin{tabular}{|c|c|c|c|c|c|}
\hline \multirow{3}{*}{8} & $Q_{s}$ & - & - & 48 & 72 \\
\cline { 2 - 6 } & \multirow{2}{*}{$Q_{r}$} & & & 38 & 60 \\
& & & & 36 & 56 \\
\hline
\end{tabular}

Table (A-6) stator slot filling factor $\left(K_{\text {fil }}\right)$

\begin{tabular}{|l|l|}
\hline$K_{\text {fil }}=0.35 \rightarrow 0.4$ & For machines less than $10 \mathrm{kw}$ \\
\hline$K_{\text {fil }}=0.4 \rightarrow 0.44$ & For machines above $10 \mathrm{kw}$ \\
\hline
\end{tabular}

Table- A-7: end-ring length as a function of poles number and coil span $\left(y_{s c}\right)$

\begin{tabular}{|c|ll|}
\hline Poles number (2P) & \multicolumn{2}{|l|}{ End turn $\left(\ell_{e t}\right)$ as a function of coil span } \\
\hline 2 & $\ell_{e t}=\left(2 * y_{s c}-0.04\right), y_{s c}$ & in meter \\
\hline 4 & $\ell_{e t}=\left(2 * y_{s c}-0.02\right), y_{s c}$ & in meter \\
\hline 6 & $\ell_{e t}=\left(\frac{\pi}{2} * y_{s c}+0.018\right), y_{s c}$ & in meter \\
\hline 8 & let $=\left(2.2 * y_{s c}-0.012\right), y_{s c}$ & in meter \\
\hline
\end{tabular}

Table- A-8: The factor $\left(\mathrm{Y}_{d s}\right)$ as a function of number of slots / pole / phase $\left(q_{s}\right)$

\begin{tabular}{|c|l|}
\hline$q_{s}$ & $\begin{array}{l}\text { The factor } \gamma_{d s} \text { in the equation of stator slot differential } \\
\text { permeance }\end{array}$ \\
\hline 1 & $\gamma_{d s}=9.5 * 10^{-2}$ \\
\hline 2 & $\gamma_{d s}=(0.25 * \sin (\theta)+2.6) * 10^{-2}$ \\
\hline 3 & $\gamma_{d s}=(0.18 * \sin (\theta)+1.24) * 10^{-2}$ \\
\hline 4 & $\gamma_{d s}=(0.24 * \sin (\theta)+0.76) * 10^{-2}$ \\
\hline 6 & $\gamma_{d s}=(0.11 * \sin (\theta)+0.41) * 10^{-2}$ \\
\hline
\end{tabular}

Table- A-9: Carter coefficient $\left(K_{c a}\right)$

\begin{tabular}{|l|l|}
\hline$K_{c a}=1.2-1.3$ & For semi-closed stator slots \\
\hline$K_{c a}=1.5-1.7$ & For open-stator slots \\
\hline
\end{tabular}

Table- A-10: Machine mechanical losses $\left(P_{m e c}\right)$ as a function of pole-pairs

\begin{tabular}{|c|l|}
\hline pole-pairs $(\mathrm{P})$ & $\begin{array}{l}\text { Mechanical losses }\left(P_{\text {mec }}\right) \text { in terms of } \\
P_{\text {out }}\end{array}$ \\
\hline 1 & $P_{\text {mec }}=0.03 * P_{\text {out }}$ \\
\hline 2 & $P_{\text {mec }}=0.012 * P_{\text {out }}$ \\
\hline 3,4 & $P_{\text {mec }}=0.008 * P_{\text {out }}$ \\
\hline
\end{tabular}

Table- A-11: temperature convection coefficient $\left(\propto_{\text {conv }}\right)$ as a function of poles number

\begin{tabular}{|c|c|}
\hline Poles number (2P) & $\propto_{\text {conv }}\left(\mathrm{W} / \mathrm{m}^{2} . \mathrm{K}\right)$ \\
\hline 2 & 60 \\
\hline 4 & 50 \\
\hline 6 & 40 \\
\hline 8 & 32 \\
\hline
\end{tabular}

\section{REFERENCES}

1. A.K. Al-Jabri and A.I. Alolah," Limits on the performance of the three-phase self-excited induction generators ", IEEE Transactions on Energy Conversion, Vol. 5, No. 2, Pp 350-356, June 1990.

2. B. Sawetsakulanond, P. Hothongkham, and V. Kinnares, " Investigation on the performance between standard and highefficiency induction machines operating as grid-connected induction generator ", IEEE ICSET, Pp 949-953, 2008.

3. B. A. Nasir," Modeling of a self-excited induction generator in the synchronously rotating frame including dynamic saturation and iron core loss into account ", International Journal of Electrical and Computer Science, Vol. 20, No. 1, Pp 1-6, February 2020.

4. B. A. Nasir," and R.W. Daoud, " Modeling of wind turbine - selfexcited induction generator system with pitch angle and excitation capacitance control ", AIP Conference Proceedings, Vol. 2307, No. 1, Pp 1-21, December 2020.

5. B. A. Nasir, " An accurate iron core loss model in the equivalent circuit of induction machines ", Journal Of Energy, Hindawi Publisher, Vol . 2020, Article ID 7613737, Pp 1-10, 2020. 
6. J. Faiz, A. A. Dadgari, S. Horning and A. Keyhani, " Design of a three-phase self-excited induction generator ", IEEE Transactions on Energy Conversion, Vol. 10, No. 3 Pp 516-52, 1995.

7. B. Sawetsakulanond, P. Hothongkham, and V. Kinnares, " Design and construction of three-phase of self-excited induction generator ", IEEE ICSET, Pp 1373-1378, 2008.

8. Ion Boldea, " Wound-rotor induction generators: Design and testing: Variable speed generators ", Book, CRC Press, 6 October 2015.

9. B. Kareem, T. Ewetumo, M.K. Adeyeri, A. Oyetunji, and S.T. Olowookere, " Development of electricity generating system for a micro-power plant ", Journal of Production Engineering, Vol. 21, No. 2, Pp 43-49, 2018.

10. A.G. Yetgin, M. Turan, B. Cevher, A.I. Canakoglu, and A. Gun, " Induction motor design process and the effect of output coefficient ", $7^{\text {th }}$ International Conference on Advanced Technologies (ICAT ' 18), Pp 152-159, April / May 2018.

11. P.S. Prakash, " Multi-objective design of induction motor using artificial intelligence techniques ", International Research Journal Of Engineering Technology (IRJET), Vol. 4, No. 7, Pp 3529-3535, July 2017.

12. P.S. Prakash, " Elegant design strategy for multi-objective optimization of induction motors ", International Research Journal of Engineering and Technology (IRJET), Vol. 4, No. 8, Pp 2395-2401, August 2017.

13. B. Alizadeh and S.A. Gholamian, " Application of Ant colony optimization algorithm for the optimal design of squirrel cage induction motor ", International Journal of Mechatronic, Electrical and Computer Technology, Vol. 4, No. 13, Pp 1674-1690, October 2014.

14. I. Boldea and S.A. Nasar, " The induction machines design handbook ", Second Edition, Taylor and Frances Group, CRC Press, 2010.

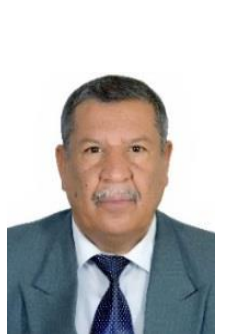

\section{AUTHORS PROFILE}

Bilal A. Nasir , (Non-member) He was born in 1958, Iraq. He received B.Sc. of electrical engineering from university of Technology, Baghdad-Iraq, 1980, and M.Sc. of electrical engineering from university of Mosul, Iraq, in 1984 and PH.D. of electrical engineering from Al-Mustansiriyah university, Iraq, in 1997. He has been employed as a lecturer in Northern Technical University ,Iraq /Hawijah Technical Institute, Kirkuk, Iraq from 1988 up to date. Now he was an assistant professor in electrical power and machines.

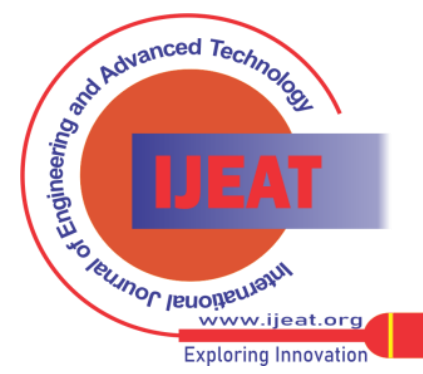

